\title{
Sufficient conditions on Liouville type theorems for the 3D steady Navier-Stokes equations
}

\author{
G. Seregin $* \dagger$ W. Wang ${ }^{\ddagger}$
}

May 8, 2018

\begin{abstract}
Our aim is to prove Liouville type theorems for the three dimensional steadystate Navier-Stokes equations provided the velocity field belongs to some Lorentz spaces. The corresponding statement contains several known results as a particular case.
\end{abstract}

Keywords: Liouville theorem, Navier-Stokes equations, Lorentz spaces

\section{Introduction}

The classical Liouville type problem is to describe all bounded solutions to the three dimensional steady-state Navier-Stokes equations

$$
-\Delta u+u \cdot \nabla u=-\nabla p, \quad \operatorname{div} u=0
$$

in the entire space $\mathbb{R}^{3}$. This is still an open problem.

Another Liouville type problem is to show that all solutions to system (1.1) belonging to the space $\stackrel{\circ}{J}_{2}^{1}$, which is the closure of the set of all smooth divergence free compactly supported functions, denoted by $C_{0,0}^{\infty}\left(\mathbb{R}^{3}\right)$, with respect to the semi-norm

$$
\|\nabla u\|_{L_{2}\left(\mathbb{R}^{3}\right)}=\left(\int_{\mathbb{R}^{3}}|\nabla u|^{2} d x\right)^{\frac{1}{2}},
$$

are identically equal to zero. This problem is related to the name of J. Leray and, to the best of authors's knowledge, has not been solved yet.

However, there are several sufficient conditions providing that all solutions $u$ to (1.1) are equal zero. Let us list the most interesting ones.

* Oxford University, UK

${ }^{\dagger}$ PDMI, RAS, Russia

${ }^{\ddagger}$ Dalian University of Technology, China 
We start with Galdi's result. Galdi proved the above Liouville type theorem under the assumption that

$$
u \in L^{\frac{9}{2}}\left(R^{3}\right)
$$

in [5]. Another interesting result belongs to Chae. In [1], he showed the condition

$$
\triangle u \in L^{\frac{6}{5}}\left(R^{3}\right)
$$

is sufficient for $u \equiv 0$ in $\mathbb{R}^{3}$. Also, Chae-Wolf gave a logarithmic improvement of Galdi's result in [3], assuming that

$$
N(u):=\int_{\mathbb{R}^{3}}|u|^{\frac{9}{2}}\{\ln (2+1 /|u|)\}^{-1} d x<\infty .
$$

Let us notice two other sufficient conditions. It has been shown in [8] that the condition

$$
u \in B M O^{-1}\left(\mathbb{R}^{3}\right)
$$

implies $u \equiv 0$ as well. Moreover, Kozono, et al., proved in [7] that $u \equiv 0$ if the vorticity

$$
w=o\left(|x|^{-\frac{5}{3}}\right)
$$

for sufficiently large $|x|$ or

$$
\|u\|_{L^{\frac{9}{2}, \infty}\left(\mathbb{R}^{3}\right)} \leq \delta D(u)^{1 / 3}
$$

for a small constant $\delta$. More references, we refer to [4, 6, 9] and the references therein.

One of our aims is to relax the restriction imposed on the norm $\|u\|_{L^{\frac{9}{2}, \infty}\left(\mathbb{R}^{3}\right)}$ in [7]. Let us remind the definition of the Lorentz spaces.

Suppose that $\Omega \subseteq \mathbb{R}^{n}$ and $1 \leq p<\infty, 1 \leq \ell \leq \infty$. It is said that a measurable function $f$ belongs to the Lorentz space $L^{p, \ell}(\Omega)$ if $\|f\|_{L^{p, \ell}(\Omega)}<+\infty$, where

$$
\|f\|_{L^{p, \ell}(\Omega)}:=\left\{\begin{array}{l}
\left(\int_{0}^{\infty} \sigma^{\ell-1}|\{x \in \Omega:|f(x)|>\sigma\}|^{\frac{\ell}{p}} d \sigma\right)^{\frac{1}{\ell}} \quad \text { if } \ell<+\infty, \\
\sup _{\sigma>0} \sigma|\{x \in \Omega:|f(x)|>\sigma\}|^{\frac{1}{p}} \quad \text { if } \ell=+\infty .
\end{array}\right.
$$

Given $u$, define the following quantity

$$
M_{\gamma, q, \ell}(R):=R^{\gamma-\frac{3}{q}}\|u\|_{L^{q, \ell}\left(B_{R} \backslash B_{R / 2}\right)}
$$

where $B(R)=B(0, R)$.

Our result is as follows:

Theorem 1.1. Let $u$ and $p$ be a smooth solution to (1.1).

(i) For $q>3,3 \leq \ell \leq \infty$ (or $q=\ell=3), \gamma=\frac{2}{3}$, assume that

$$
\liminf _{R \rightarrow \infty} M_{\frac{2}{3}, q, \ell}(R)<\infty
$$


then

$$
D(u):=\int_{\mathbb{R}^{3}}|\nabla u|^{2} d x \leq c(q, \ell) \liminf _{R \rightarrow \infty} M_{\frac{2}{3}, q, \ell}^{3}(R) .
$$

Moreover, if

$$
\liminf _{R \rightarrow \infty} M_{\frac{2}{3}, q, \ell}^{3}(R) \leq \delta D(u)
$$

for some $0<\delta<1 / c(q, \ell)$, then $u \equiv 0$.

(ii) For $12 / 5<q<3,1 \leq \ell \leq \infty, \gamma>\frac{1}{3}+\frac{1}{q}$, suppose that

$$
\liminf _{R \rightarrow \infty} M_{\gamma, q, \ell}(R)<\infty
$$

holds then $u \equiv 0$ as well.

Remarks 1.2. (i) Letting $q=\ell=\frac{9}{2}$ and assuming that $u \in L_{\frac{9}{2}}\left(\mathbb{R}^{3}\right)$, we observe that $M_{\frac{2}{3}, \frac{9}{2}, \frac{9}{2}}(R) \rightarrow 0$ as $R \rightarrow \infty$. So, Galdi's result follows from Theorem 1.1 .

(ii) For $q=\frac{9}{2}$ and $\ell=\infty$, condition (1.4) can be regarded as a generalisation a result proved by Kozono-Terasawa-Wakasugi in [7].

(iii) If we let $N(u)=\infty$, then $N(v)=\infty$ for the function $v=|x|^{-\frac{2}{3}}[\ln \ln (|x|+e)]^{-\nu}$ with $0<\nu \leq \frac{2}{9}$. However, if we assume that

$$
|u| \leq \frac{C}{|x|^{\frac{2}{3}}[\ln \ln (|x|+e)]^{\nu}}
$$

for the same $\nu$, we can easily check the following fact

$$
\|u\|_{L^{\frac{9}{2}, \infty}(B(R) \backslash B(R / 2))} \rightarrow 0
$$

as $R \rightarrow \infty$. The latter shows that statement (i) of Theorem 1.1 does not follow from the result of Chae-Wolf [3].

(iii) The second statement of the theorem is an improvement of one of the results in [10], see Theorem 1.8, where it is assume that $\gamma>\frac{4 q-3}{6 q-3}$.

\section{Caccioppoli Type Inequalities}

We start with an auxiliary lemma about Caccioppoli type inequality for the system (1.1).

Proposition 2.1. Let $u$ and $p$ be the smooth solution of (1.1). Then the following Caccioppoli type inequalities hold:

if $q>3$ and $1 \leq \ell \leq \infty$, then

$$
\int_{B(R / 2)}|\nabla u|^{2} d x \leq C R^{-2} \int_{B(R) \backslash B(R / 2)}|u|^{2} d x+C R^{2-\frac{9}{q}}\|u\|_{L^{q, \ell}(B(R) \backslash B(R / 2))}^{3} ;
$$


if $0<\delta \leq 1,3>q>\frac{6(3-\delta)}{6-\delta}$, then

$$
\begin{gathered}
\int_{B(R / 2)}|\nabla u|^{2} d x \leq \frac{C}{R^{2}} \int_{B(R) \backslash B(R / 2)}|u|^{2} d x+ \\
+C(\delta)\left(\|u\|_{L^{q, \infty} 2(B(R) \backslash B(R / 2))}^{3-\delta} R^{2-\frac{9-3 \delta}{q}-\frac{\delta}{2}}\right)^{\frac{2}{2-\delta}} .
\end{gathered}
$$

Proof. Given $R>0$, fix numbers $\varrho$ and $r$ so that $3 R / 4 \leq \varrho<r \leq R$. Now, let us pick up a cut-off function $\phi(x) \in C_{0}^{\infty}(B(R))$ satisfying the following conditions: $0 \leq \phi \leq 1$, $\phi(x)=1$ if $x \in B(\varrho), \phi(x)=0$ if $x \in B(r)^{c}$, and $|\nabla \phi(x)| \leq c /(r-\varrho)$.

We also may assume that function $\phi(x)=\eta(|x|)$, i.e., it depends on the distance to the origin only. In this case, it is easy to check that

$$
\int_{B(r) \backslash B(2 r / 3)} \nabla \phi \cdot u d x=0 .
$$

Then, by Theorem III 3.4 in [5] and by scaling, for any $1<s<\infty$, there exist a constant $c_{0}(s)$ and a function $w \in W_{s}^{1}(B(r))$ such that div $w=\nabla \phi \cdot u$ in $B(r), w=0$ on $\partial B(r) \cup \partial B(2 r / 3)$, and

$$
\int_{B(r) \backslash B(2 r / 3)}|\nabla w|^{s} d x \leq C_{0}(s) \int_{B(r) \backslash B(2 r / 3)}|\nabla \phi \cdot u|^{s} d x .
$$

The function $w$ is extended by zero outside the set $B(r) \backslash B(2 r / 3)$. Moreover, it is actually smooth as $u$ is smooth.

According to the general Marcinkiewicz interpolation theorem, we find

$$
\|\nabla w\|_{L^{q, \ell}(B(r) \backslash B(2 r / 3))} \leq C_{0}(q)\|\nabla \phi \cdot u\|_{L^{q, \ell}(B(r) \backslash B(2 r / 3))} .
$$

for any $1<q<\infty$ and any $1 \leq \ell \leq \infty$.

Multiplication of both sides of the equation (1.1) by $(\phi u-w)$ and integration by parts give:

$$
\begin{gathered}
\int_{B(r)} \phi|\nabla u|^{2} d x= \\
=-\int_{B(r)} \nabla u:(\nabla \phi \otimes u) d x+\int_{B(r)} \nabla w: \nabla u d x-\int_{B(r)} \nabla u:(\phi u \otimes u) d x+ \\
+\int_{B(r)} \nabla u:(u \otimes w) d x=I_{1}+\cdots+I_{4} .
\end{gathered}
$$


Obviously, since $R \geq r>\varrho \geq 3 R / 4>R / 2$,

$$
\begin{gathered}
\left|I_{1}\right| \leq C \frac{1}{r-\rho}\left(\int_{B(r)}|\nabla u|^{2} d x\right)^{\frac{1}{2}}\left(\int_{B(r) \backslash B(\varrho)}|u|^{2} d x\right)^{\frac{1}{2}} \leq \\
C \frac{1}{r-\rho}\left(\int_{B(r)}|\nabla u|^{2} d x\right)^{\frac{1}{2}}\left(\int_{B(R) \backslash B(R / 2)}|u|^{2} d x\right)^{\frac{1}{2}}
\end{gathered}
$$

and

$$
\begin{aligned}
\left|I_{2}\right| & \leq C\left(\int_{B(r)}|\nabla u|^{2} d x\right)^{\frac{1}{2}}\|\nabla w\|_{L_{2}(B(r) \backslash B(2 r / 3))} \leq \\
& \leq C \frac{1}{r-\rho}\|\nabla u\|_{L_{2}(B(r))}\|u\|_{L_{2}(B(R) \backslash B(R / 2))} .
\end{aligned}
$$

Now, our aim is to prove inequality (2.1). To this end, assuming that $q>3$ and $\ell \geq 3$, let us estimate $I_{3}$, using integration by parts and Hölder inequality in Lorentz spaces. Indeed,

$$
\begin{gathered}
\left|I_{3}\right|=\left.\frac{1}{2}\left|\int_{B(r) \backslash B(\varrho)} u \cdot \nabla \phi\right| u\right|^{2} d x \mid \leq \\
\leq C\|u \cdot \nabla \phi\|_{L^{\frac{q}{q-2},}, \frac{\ell}{\ell-2}(B(r) \backslash B(\varrho))}\left\||u|^{2}\right\|_{L^{\frac{q}{2}, \frac{\ell}{2}}(B(r) \backslash B(\varrho))} \leq \\
\leq C \frac{1}{r-\varrho}\|u\|_{L^{q, \ell}(B(R) \backslash B(R / 2))}^{3}\|1\|_{L^{\frac{q}{q-3}, \frac{\ell}{\ell-3}}(B(R) \backslash B(R / 2))} \leq \\
\leq C \frac{1}{r-\rho}\|u\|_{L^{q, \ell}(B(R) \backslash B(R / 2))}^{3} R^{3-\frac{9}{q}} .
\end{gathered}
$$

The quantity $I_{4}$ is evaluated similarly, if we use the estimate for the gradient of $w$ with suitable exponents:

$$
\begin{gathered}
\left|I_{4}\right|=\left|\int_{B(r) \backslash B(2 r / 3)} \nabla w: u \otimes u d x\right| \leq \\
\leq C\|\nabla w\|_{L^{\frac{q}{q-2}, \frac{\ell}{\ell-2}}(B(r) \backslash B(2 r / 3))}\left\||u|^{2}\right\|_{L^{\frac{q}{2}, \frac{\ell}{2}}(B(r) \backslash B(2 r / 3)} \leq \\
\leq C\|u \cdot \nabla \phi\|_{L^{\frac{q}{q-2}}, \frac{\ell}{\ell-2}(B(r) \backslash B(2 r / 3))}\|u\|_{L^{q, \ell}(B(r) \backslash B(2 r / 3))}^{2} \leq \\
\leq \frac{C}{\tau-\rho}\|u\|_{L^{q, \ell}(B(R) \backslash B(R / 2))}^{3} R^{3-\frac{9}{q}} .
\end{gathered}
$$

Hence, we get

$$
\begin{aligned}
\int_{B(\rho)}|\nabla u|^{2} d x \leq & \frac{1}{2} \int_{B(r)}|\nabla u|^{2} d x+\frac{C}{(r-\rho)^{2}}\left(\int_{B(R) \backslash B(R / 2)}|u|^{2} d x\right)+ \\
& +\frac{C}{r-\rho}\|u\|_{L^{q, \ell}(B(R) \backslash B(R / 2))}^{3} R^{3-\frac{9}{q}},
\end{aligned}
$$


which yields the inequality (2.1) by the standard iteration.

Now, let us prove the second inequality of the proposition. To this end, we introduce $\bar{u}=u-[u]_{B(r) \backslash B(2 r / 3)}$, where $[u]_{\Omega}$ is the mean value of $u$ over a domain $\Omega$. Applying integration by parts, we find

$$
\begin{gathered}
I_{3}=-\frac{1}{2} \int_{B(r)} \phi u \cdot \nabla\left(|u|^{2}\right) d x=-\frac{1}{2} \int_{B(r)} \phi u \cdot \nabla\left(|u|^{2}-\left|[u]_{B(r) \backslash B(2 r / 3)}\right|^{2}\right) d x= \\
=\frac{1}{2} \int_{B(r) \backslash B(\varrho)}(u \cdot \nabla \phi)\left(|u|^{2}-\left|[u]_{B(r) \backslash B(2 r / 3)}\right|^{2}\right) d x
\end{gathered}
$$

and, since $2 r / 3<3 R / 4 \leq \varrho$,

$$
\left|I_{3}\right| \leq \frac{C}{r-\varrho} \int_{B(r) \backslash B(2 r / 3)}|u||\bar{u}|\left|u+[u]_{B(r) \backslash B(2 r / 3)}\right| d x .
$$

Under our assumptions on numbers $q$ and $\delta$, the following is true

$$
0<\beta=1-\frac{3-\delta}{q}-\frac{\delta}{6}<1 .
$$

So, applying the Hölder inequality for Lorentz spaces, we show

$$
\begin{gathered}
\left|I_{3}\right| \leq \frac{C}{r-\varrho} \int_{B(r) \backslash B(2 r / 3)}|u||\bar{u}|^{1-\delta}|\bar{u}|^{\delta}\left|u+[u]_{B(r) \backslash B(2 r / 3)}\right| d x \leq \\
\leq \frac{C}{r-\varrho}\|u\|_{L^{q, \infty}(B(r) \backslash B(2 r / 3))}\left\||\bar{u}|^{(1-\delta)}\right\|_{L^{\frac{q}{1-\delta}, \infty}(B(r) \backslash B(2 r / 3))}\left\||\bar{u}|^{\delta}\right\|_{L_{\frac{6}{\delta}}(B(r) \backslash B(2 r / 3))} \times \\
\times\|1\|_{L^{\frac{1}{\beta}, \frac{6}{6-\delta}(B(r) \backslash B(2 r / 3))}}\left\|u+[u]_{B(r) \backslash B(2 r / 3)}\right\|_{L^{q, \infty}(B(r) \backslash B(2 r / 3))} \leq \\
\leq \frac{C}{r-\varrho}\|u\|_{L^{q, \infty}(B(r) \backslash B(R / 2))}\|\bar{u}\|_{L^{q, \infty}(B(r) \backslash B(2 r / 3))}^{1-\delta}\|\bar{u}\|_{L_{6}(B(r) \backslash B(2 r / 3))}^{\delta} \times \\
\times R^{3 \beta}\left\|u+[u]_{B(r) \backslash B(2 r / 3)}\right\|_{L^{q, \infty}(B(r) \backslash B(2 r / 3))} .
\end{gathered}
$$

By Gagliardo-Nireberg-Sobolev inequality and by the inequality

$$
\left\|[u]_{B(r) \backslash B(2 r / 3)}\right\|_{L^{q, \infty}(B(r) \backslash B(2 r / 3))} \leq c\|u\|_{L^{q, \infty}(B(r) \backslash B(2 r / 3))},
$$

we can transform the estimate of $\left|I_{3}\right|$ to the following final form

$$
\begin{gathered}
\left|I_{3}\right| \leq \frac{C}{r-\varrho} R^{3 \beta}\|u\|_{L^{q, \infty}(B(r) \backslash B(R / 2))}^{3-\delta}\|\nabla u\|_{L_{2}(B(r) \backslash B(2 r / 3))}^{\delta} \leq \\
\leq \frac{C}{r-\varrho} R^{3 \beta}\|u\|_{L^{q, \infty}(B(r) \backslash B(R / 2))}^{3-\delta}\|\nabla u\|_{L_{2}(B(r) \backslash B(R / 2))}^{\delta} \leq
\end{gathered}
$$




$$
\leq \frac{1}{9} \int_{B(r) \backslash B(R / 2)}|\nabla u|^{2} d x+C(\delta)\left(\frac{R^{3 \beta}}{r-\varrho}\|u\|_{L^{q, \infty}(B(r) \backslash B(R / 2))}^{3-\delta}\right)^{\frac{2}{2-\delta}} .
$$

Now, our aim is to evaluate $I_{4}$. Using similar arguments, we have

$$
\begin{gathered}
\left|I_{4}\right|=\left|\int_{B(r) \backslash B(2 r / 3)}(u \cdot \nabla u) \cdot w d x\right|=\left|\int_{B(r) \backslash B(2 r / 3)}(u \cdot \nabla w) \cdot \bar{u} d x\right| \leq \\
\leq\|\nabla w\|_{L^{q, \infty}(B(r) \backslash B(2 r / 3))}\|\bar{u}\|_{L^{q, \infty}(B(r) \backslash B(2 r / 3))}^{1-\delta}\|\bar{u}\|_{L_{6}(B(r) \backslash B(2 r / 3))}^{\delta} \times \\
\times R^{3 \beta}\|u\|_{L^{q, \infty}(B(r) \backslash B(2 r / 3))} .
\end{gathered}
$$

Taking into account the bound for the gradient of $w$, we arrive at the same type estimate as in the case of $I_{3}$

Consequently, combining bounds of $I_{1}, \cdots, I_{4}$ we get

$$
\begin{gathered}
\int_{B(\varrho)}|\nabla u|^{2} d x \leq \frac{1}{2} \int_{B(r)}|\nabla u|^{2} d x+\frac{C}{(r-\rho)^{2}}\left(\int_{B(R) \backslash B(R / 2)}|u|^{2} d x\right) \\
+C(\delta)\left(\frac{R^{3 \beta}}{(r-\varrho)}\|u\|_{L^{q, \infty}(B(R) \backslash B(R / 2))}^{3-\delta}\right)^{\frac{2}{2-\delta}}
\end{gathered}
$$

for any $\frac{3}{4} R \leq \varrho<\tau \leq R$. Hence, the inequality (3.1) follows.

\section{Proof of Theorem 1.1}

We start with a proof of the statement (i). It is easy to check that, for $2<q<6$, the following estimate is valid:

$$
\begin{gathered}
R^{-2}\left(\int_{B(R) \backslash B(R / 2)}|u|^{2} d x\right) \leq C(q) R^{1-\frac{6}{q}}\|u\|_{L^{q, \infty}(B(R) \backslash B(R / 2))}^{2} \leq \\
\leq C(q) R^{-\frac{1}{3}} M_{\frac{2}{3}, q, \ell}^{2}(R) .
\end{gathered}
$$

Taking into account condition (1.2), we find (1.3) and then (1.4).

Now, our goal is to prove the statement (ii). Applying the Hölder inequality to the first term on the right hand side in (3.1), we find the following:

$$
\begin{gathered}
\int_{B(R / 2)}|\nabla u|^{2} d x \leq C R^{\frac{1}{3}-\frac{2}{q}} M_{\frac{1}{3}+\frac{1}{q}, q, \infty}^{2}(R)+C(\delta)\left(\|u\|_{L^{q, \infty} 2(B(R) \backslash B(R / 2))}^{3-\delta} R^{3 \beta-1}\right)^{\frac{2}{2-\delta}} \leq \\
\leq C R^{\frac{1}{3}-\frac{2}{q}} M_{\frac{1}{3}+\frac{1}{q}, q, \infty}^{2}(R)+C\left(M_{\gamma, q, \infty}^{3-\delta}(R) R^{3 \beta-1-\left(\gamma-\frac{3}{q}\right)(3-\delta)}\right)^{\frac{2}{2-\delta}}= \\
=C R^{\frac{1}{3}-\frac{2}{q}} M_{\frac{1}{3}+\frac{1}{q}, q, \infty}^{2}(R)+C\left(M_{\gamma, q, \infty}^{3-\delta}(R) R^{2-\frac{\delta}{2}-\gamma(3-\delta)}\right)^{\frac{2}{2-\delta}} .
\end{gathered}
$$


Now, fix $q \in] 12 / 5,3\left[\right.$. Then we can find $q_{1}$ having the following properties:

$$
q>q_{1}>\frac{12}{5}, \quad \gamma>\frac{1}{3}+\frac{1}{q_{1}}>\frac{1}{3}+\frac{1}{q} .
$$

Given $q_{1}$, there exists a number $\left.\delta \in\right] 0,1[$ such that

$$
q_{1}=\frac{6(3-\delta)}{6-\delta}<q
$$

It remains to notice that

$$
\begin{aligned}
a:=2-\frac{\delta}{2}-\gamma(3-\delta) & =2-\frac{3\left(3-q_{1}\right)}{6-q_{1}}-\gamma\left(3-\frac{6\left(3-q_{1}\right)}{6-q_{1}}\right)= \\
& =\frac{3+q_{1}-3 q_{1} \gamma}{6-q_{1}} .
\end{aligned}
$$

But $\gamma>\frac{1}{3}+\frac{1}{q_{1}}$ and thus

$$
a=3 q_{1} \frac{\frac{1}{3}+\frac{1}{q}{ }_{1}-\gamma}{6-q_{1}}<0
$$

Passing to the limit as $R \rightarrow \infty$, we complete the proof of the theorem.

Acknowledgments. The first author is supported by the grant RFBR 17-01- 00099-a. W. Wang was supported by NSFC under grant 11671067, "the Fundamental Research Funds for the Central Universities" and China Scholarship Council.

\section{References}

[1] D. Chae, Liouville-type theorem for the forced Euler equations and the NavierStokes equations. Commun. Math. Phys.326: 37-48 (2014).

[2] D. Chae, T. Yoneda, On the Liouville theorem for the stationary Navier-Stokes equations in a critical space, J. Math. Anal. Appl. 405 (2013), no. 2, 706-710.

[3] D. Chae, J. Wolf, On Liouville type theorems for the steady Navier- Stokes equations in $R^{3}$, arXiv:1604.07643.

[4] Chae, G., Weng, S., Liouville type theorems for the steady axially symmetric Navier-Stokes and magnetohydrodynamic equations, Discrete And Continuous Dynamical Systems, Volume 36, Number 10, 2016 Pp. 5267-5285.

[5] G. P. Galdi, An introduction to the mathematical theory of the Navier- Stokes equations. Steady-state problems. Second edition. Springer Monographs in Mathematics. Springer, New York, 2011. xiv+1018 pp.

[6] Koch, G., Nadirashvili, N., Seregin, G., Sverak, V., Liouville theorems for the Navier-Stokes equations and applications, Acta Mathematica, 203 (2009), 83105. 
[7] H. Kozono, Y. Terasawab, Y. Wakasugib, A remark on Liouville-type theorems for the stationary NavierCStokes equations in three space dimensions, Journal of Functional Analysis, 272(2017), 804-818.

[8] G. Seregin, Liouville type theorem for stationary Navier-Stokes equations, Nonlinearity, 29 (2016), 2191-2195.

[9] G. Seregin, A liouville type theorem for steady-state Navier-Stokes equations, arXiv:1611.01563 and J. E. D. P. (2016), Expos no IX, 5 p.

[10] G. Seregin, Remarks on Liouville type theorems for steady-state Navier-Stokes equations, arXiv:1703.10822v1 and Algebra i Analiz, 2018, Vol. 30, no.2., 238248. 